\title{
Le croissant culturel et touristique de Chicoutimi : une démarche de revitalisation par le biais des arts et de la culture ${ }^{1}$
}

\author{
Martin Simard ${ }^{2}$ et Nadine Maltais ${ }^{3}$
}

\section{Introduction}

Comme la plupart des villes moyennes nordaméricaines, la ville regroupée de Saguenay possède des rues commerciales traditionnelles qui ont été affectées par la montée des centres d'achats périurbains ${ }^{4}$. En plus de la fonction commerciale, ces centres-villes rassemblent pourtant des activités portuaires et industrielles ainsi que des espaces publics et des équipements institutionnels, ce qui leur procure des avantages de locali-

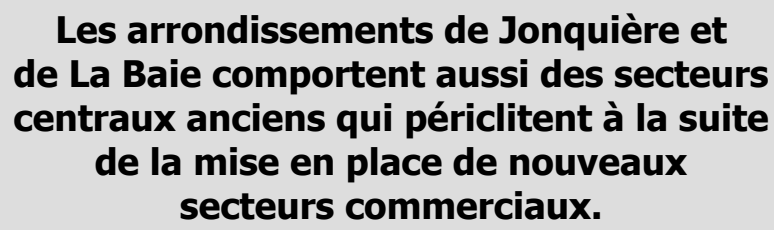

tage les pôles périphériques ${ }^{7}$. L'habitat limitrophe montre aussi des signes de dépérissement. Celui-ci est caractérisé par des duplex et des triplex isolés qui accueillent des populations plutôt défavorisées ${ }^{8}$. Néanmoins, le centre historique de l'arrondissement de Chicoutimi demeure une aire de concentration d'activités de bureau, plus spécialement pour le tertiaire public municipal et provincial. D'autre part, on y remarque des équipements collectifs d'envergure tels que le site de la Pulperie, le parc de la zone portuaire, la bibliothèsation significatifs ${ }^{5}$. Ce mouvement de désaffection touche notamment le centre historique de l'ancienne ville de Chicoutimi, laquelle constitue maintenant l'un des trois arrondissements de Saguenay. À Chicoutimi, la ville nouvelle s'est établie sur les plateaux périphériques de la plaine du Haut-Saguenay, rendant presque marginale la ville ancienne localisée en contrebas, sur les rives de la rivière Saguenay. Les arrondissements de Jonquière et de La Baie comportent aussi des secteurs centraux anciens qui périclitent à la suite de la mise en place de nouveaux secteurs commerciaux. Ce contexte contribue à façonner une dynamique urbaine diffuse et fragmentée à Saguenay ${ }^{6}$.

Au cours du dernier quart de siècle, le secteur de la rue Racine à Chicoutimi a subi une décroissance importante qui semble directement proportionnelle à l'expansion de l'axe d'affaires du boulevard Talbot. Ce déclin s'affiche sur le tissu commercial tant au plan quantitatif que qualitatif, même si l'on remarque une stabilisation de la situation. Le déménagement récent d'un concessionnaire automobile illustre bien la tendance lourde du développement urbain qui avan- que municipale, le CLSC, la Cathédrale, l'évêché, le conservatoire de musique et, à relative proximité, l'hôpital, le collège et l'université.

C'est sur son caractère urbain et la concentration d'activités diverses que pourrait se trouver la voie d'un nouvel élan de développement pour le centreville de Chicoutimi. En effet, les secteurs centraux traditionnels constituent des espaces de sociabilité et des lieux de mémoire qui se distinguent des espaces résidentiels et commerciaux modernes qui caractérisent la banlieue. Les quartiers récemment implantés paraissent davantage conçus pour la voiture que pour les humains malgré leur dimension fonctionnelle. D'ailleurs, le courant du nouvel urbanisme qui est présentement à l'honneur prône le rétablissement d'un certain niveau de densité et de diversité dans les projets d'aménagement afin de donner corps à la ville actuelle, laquelle se révèle aseptisée et froide à plusieurs égards ${ }^{9}$.

C'est dans ce contexte que le projet de Croissant culturel et touristique a pris forme au cœur de l'arrondissement de Chicoutimi. Celui-ci a été élaboré à travers le leadership des gens d'affaires et l'expertise 
des planificateurs municipaux. Le concept de Croissant culturel et touristique tente de tirer profit des commerces et institutions en place ainsi que des infrastructures socioculturelles et des ateliers d'artistes qui sont nombreux sur ce territoire. Les artistes se présentent fréquemment comme de véritables pionniers urbains qui investissent des quartiers symboliquement dévalorisés mais accessibles au plan économique. Ils peuvent jouer un rôle déterminant dans la revitalisation de quartiers anciens, comme ce fut le cas dans le quartier Saint-Roch à Québec ${ }^{10}$. Les artistes participent à l'occupation du territoire et à l'animation urbaine, ce qui s'avère un prélude au développement touristique du milieu, selon de multiples expériences.

Ce texte a pour but d'expliquer et de promouvoir le projet de Croissant culturel et touristique de Chicoutimi tout en identifiant les enjeux et défis liés à ce type de stratégie de revitalisation urbaine. Dans un premier temps, nous traiterons du centre-ville de Chicoutimi et des différentes démarches de revitalisation qui y ont été entreprises au fil des années. En deuxième lieu, nous décrirons l'émergence et le cadre organisationnel du projet de Croissant culturel et touristique. Finalement, nous soulèverons quelques dilemmes associés à ce programme de revitalisation par le biais des arts et de la culture.

Le concept de Croissant culturel et touristique tente de tirer profit des commerces et institutions en place ainsi que des infrastructures socioculturelles et des ateliers d'artistes qui sont nombreux sur ce territoire.

\section{La dégradation des centres-villes et les démarches de revitalisation}

\section{Le phénomène d'étalement urbain}

Le centre-ville de Chicoutimi fut pendant plus d'un siècle le point central du commerce régional et un pôle industriel, soit de la fondation de Chicoutimi en 1842 jusqu'aux années $1960{ }^{11}$. La construction du centre commercial «Place du Saguenay » en 1967, sur les abords d'un axe routier périphérique, lance un mouvement d'étalement urbain qui défavorise fortement le centre. L'étalement urbain peut être défini comme étant une expansion urbaine démesurée consécutive à la multiplication des développements domiciliaires de faible densité. À Chicoutimi, comme ailleurs au Canada et aux États-Unis, le contexte socioéconomique des années 1960 et 1970 se prêtait bien à la construction de nouveaux quartiers en fonction de règles urbanistiques standardisées ${ }^{12}$. En effet, la popularité de l'automobile, la forte croissance démographique, l'augmentation des revenus des ménages et les démarches agressives des municipalités et des promoteurs immobiliers se conjuguent pour alimenter un élan de développement rapide et vigoureux qui a été interprété comme étant de l'étalement urbain.

L'étalement urbain découle de choix individuels et familiaux qui sont rationnels, soit acquérir une propriété de qualité, à bon prix, dans un environnement calme ${ }^{13}$. Cependant, ces décisions induisent des effets néfastes lorsqu'elles sont multipliées par milliers au sein des agglomérations urbaines. À titre d'exemples, mentionnons la destruction d'écosystèmes agraires et forestiers, les émanations toxiques produites par l'utilisation excessive de l'automobile ainsi que la surconsommation d'infrastructures (routes, aqueducs, égouts, etc.) ${ }^{14}$. Pour notre propos, il faut surtout considérer que les quartiers urbains centraux sont touchés par le dépeuplement, l'appauvrissement et la dégradation physique qui découlent largement du phénomène de l'étalement urbain. En fait, on observe à cette époque un véritable transfert de populations, de commerces et d'industries du centre vers la banlieue. Cela signifie un désinvestissement et un dépérissement pour les quartiers anciens qui deviennent déclassés tant sur le marché immobilier que sur celui des valeurs culturelles. Désormais, les centres-villes ne sont plus aussi dynamiques et animés, et leur pouvoir d'attraction a fortement diminué.

\section{Le centre-ville de Chicoutimi : problématique et interventions}

Au début des années 1970, le déclin de la rue Racine amène les autorités municipales de Chicoutimi à intervenir afin de réhabiliter le centre d'affaires et les quartiers avoisinants (voir tableau 1). Les programmes de rénovation urbaine sont alors à la mode en Amérique du Nord et en Europe. Ceux-ci sont encouragés et financés en bonne partie par les gouvernements supérieurs qui aspirent à faire d'une pierre deux coups, soit à construire des autoroutes urbaines 
tout en éliminant les secteurs de taudis. Rapidement, les opérations de rénovation urbaine furent critiquées pour leurs méthodes brutales fondées sur la démolition du cadre bâti existant. Le programme de rénovation du quartier du Bassin de Chicoutimi, qui a eu lieu entre 1971 et 1976 , n'échappe pas à la règle. Des dizaines de résidences ont été détruites pour faire place au boulevard Saint-Paul qui facilitera l'accès au pont Dubuc ${ }^{15}$. L'ancien quartier ouvrier, qui devra affronter les affres du déluge en 1996, en ressort amoindri et relativement isolé du reste du centre-ville.

\section{Tableau 1 - Interventions urbanistiques majeures au centre-ville de Chicoutimi}

\begin{tabular}{cl}
\hline Date & \multicolumn{1}{c}{ Programme ou intervention } \\
$1971-1976$ & Rénovation urbaine (quartier du Bassin et l'ouest du centre-ville) \\
$1986-1987$ & Revi-centre (rue Racine) \\
$1992-1993$ & Réaménagement de la zone portuaire \\
$1996-1999$ & Programme de reconstruction lié aux inondations (quartier du Bassin) \\
$1996-2003$ & Rue principale et Réaménagement des vieux quartiers (rue Racine) \\
\hline
\end{tabular}

Au cours des années 1980 et 1990, les approches d'intervention en matière de revitalisation vont se raffiner, à Chicoutimi comme ailleurs. Celles-ci tentent de s'attaquer aux effets de milieu existant dans les quartiers en difficulté ${ }^{16}$. Ces politiques intègrent généralement un large éventail de mesures réunies autour de quatre axes: le cadre physique, l'emploi, la santé et la sécurité ${ }^{17}$. À Chicoutimi, les efforts de revitalisation se sont concentrés sur la rue Racine et la zone portuaire au cours de cette période. La rue Racine a été le théâtre de deux phases d'interventions majeures, soit le projet Revi-centre de 1986-1987 et les programmes Rue principale et Réaménagement des vieux quartiers en 1995-1996. Ces différentes démarches visaient essentiellement à dynamiser le commerce en intervenant sur les aménagements physiques (trottoirs, mobilier urbain, etc.) et auprès des commerçants, pour une promotion mieux concertée.

Les succès de ces plans d'action furent relativement limités, c'est-à-dire qu'ils ne permirent pas de renverser complètement la tendance naturelle du marché immobilier. Cependant, cela a modifié visiblement le visage du centre-ville. Il nous semble donc opportun de poursuivre les efforts en vue de rétablir un équilibre entre les centres urbains ancien et nouveau. À la lumière des expériences de revitalisation, il paraît nécessaire de concevoir de nouveaux projets à visée plus globale qui considèrent le centre-ville dans sa totalité, à la fois dans ses dimensions commerciale et institutionnelle, sans oublier son caractère résidentiel.

\section{Le Croissant culturel et touristique de l'arrondissement de Chicoutimi}

\section{Émergence et caractéristiques du projet}

En 1996, on assiste à la constitution d'un nouveau regroupement de gens d'affaires à la suite de la dissolution de la SIDAC, soit l'Association des centresvilles de Chicoutimi (ACVC) ${ }^{18}$. Dans les années qui suivent, cette association met de l'avant le concept de Croissant culturel et touristique (CCT). Dès le départ, le concept cherchait à mettre en valeur la forte présence d'organismes et d'intervenants au centre-ville de Chicoutimi dans les domaines artistique, patrimonial et touristique. Ces acteurs locaux possèdent un immense potentiel de développement dans la mesure où l'on consolide au centre-ville un environnement favorisant les échanges et les synergies. En décembre 2001, après une période de mûrissement, le Croissant culturel et touristique de Chicoutimi recevait un appui symbolique et logistique de la part du conseil municipal par l'adoption d'une résolution officialisant son existence et par l'intégration de ce concept au plan d'urbanisme de la municipalité.

Le principe de Croissant culturel et touristique s'inspire des recherches et actions du réseau québécois des 
Villes et villages d'art et de patrimoine (VVAP), réseau auquel l'Association des centres-villes s'est jointe. Ce réseau a pour mission de promouvoir les ressources culturelles des communautés locales afin de mettre en valeur les arts, la culture et le patrimoine dans une optique de développement local. Le Croissant culturel et touristique veut être un projet moteur qui rassemble et identifie les musées, ateliers et autres institutions et commerces ayant pour trait commun de faire partie de l'infrastructure culturelle et touristique du centre-ville de l'arrondissement de
Chicoutimi. Ces établissements se situent à l'intérieur d'une bande de terrain de forme elliptique s'étirant, d'est en ouest, de la Cathédrale de Chicoutimi à la Pulperie en passant par la zone portuaire, soit la majeure partie du centre-ville. Les rues Racine et Jacques-Cartier sont les deux artères commerciales principales qui traversent ce secteur. La superficie de cette zone aux frontières volontairement floues est d'environ 150 ha. (voir figure 1). Elle concentre une population de 3316 habitants ${ }^{19}$, soit près de $5 \%$ de la population de l'arrondissement de Chicoutimi.

\section{Figure 1 - Localisation du Croisant culturel et touristique de Chicoutimi}

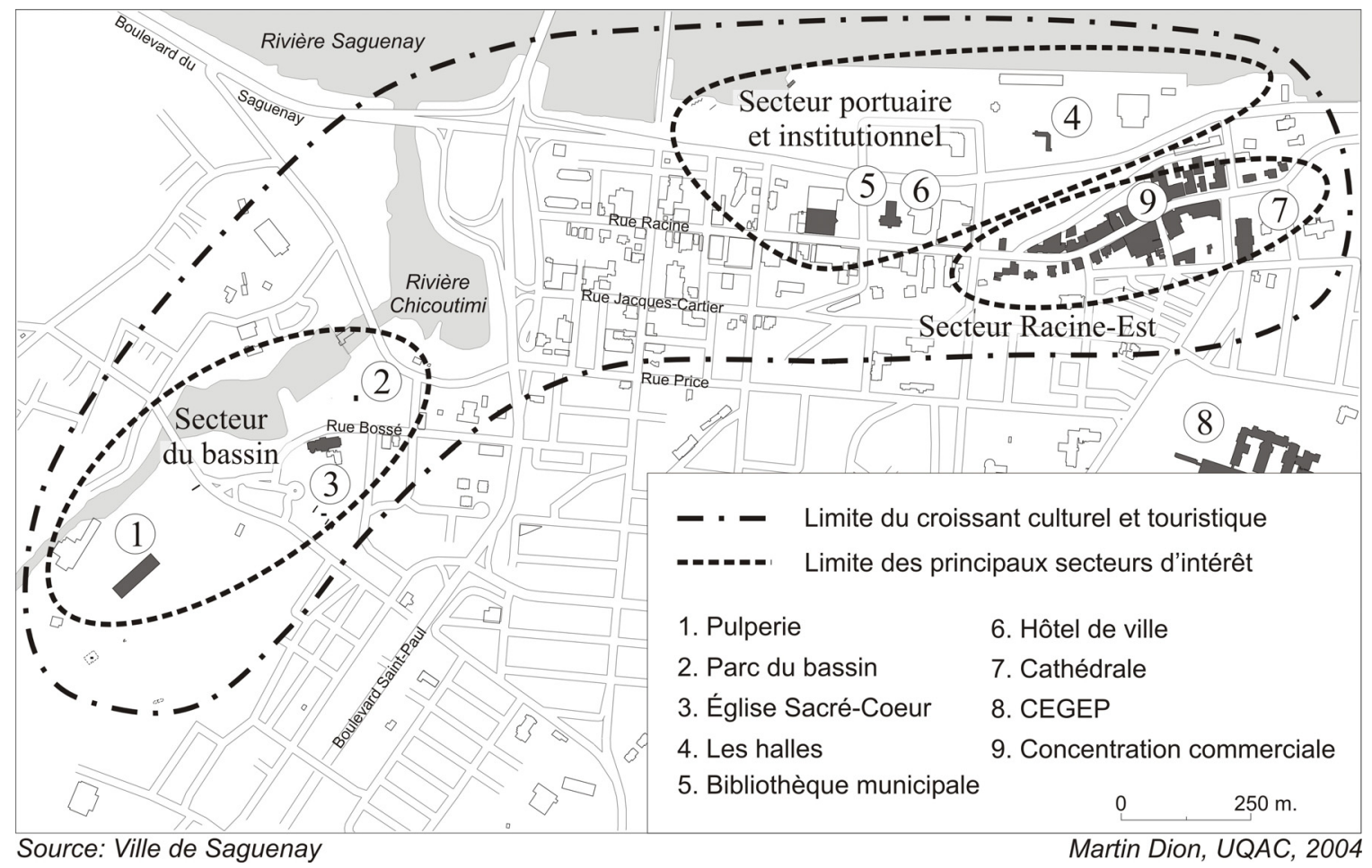

La zone délimitée par le Croissant culturel et touristique est sans aucun doute celle qui est la plus marquée par l'histoire dans l'ancienne ville de Chicoutimi. C'est au sein de cet espace au relief accidenté que la centralité urbaine a pris forme dans la deuxième moitié du $\mathrm{XIX}^{\mathrm{e}}$ siècle. On y érige le quartier institutionnel et commercial aux abords de la rivière Saguenay afin d'offrir des services aux deux noyaux villageois situés aux embouchures des rivières Chicoutimi et du Moulin ${ }^{20}$ À notre époque, on peut identifier trois secteurs disposant d'attraits importants à relative proximité à l'intérieur du territoire du Croissant. Il s'agit de la rue Racine Est, dans sa par- tie surélevée, du secteur portuaire et institutionnel ainsi que du quartier du Bassin aussi appelé paroisse Sacré-cœur ou centre-ville ouest, quoique cette dernière appellation fasse référence à un territoire plus large.

Le quartier du Bassin est particulièrement bien doté en équipements touristiques et en bâtiments patrimoniaux, malgré les séquelles associées à la rénovation urbaine et aux inondations. Sur les 155 bâtiments retenus en 1990 par l'ancienne ville de Chicoutimi pour fins de caractérisation architecturale, on en compte au moins 70 dans ce seul district ayant été le 
lieu d'érection du Poste de traite de Chicoutimi en $1676^{21}$. On constate également la présence sur ce territoire de l'imposant complexe touristique de la Pulperie de Chicoutimi qui comprend maintenant le musée régional du Saguenay-Lac-Saint-Jean. Toujours dans le même secteur, on note l'aménagement du parc du Bassin au milieu duquel trône fièrement l'emblématique petite maison blanche qui commémore la résistance aux inondations de 1996 (voir figure 2).

\section{Figure 2 - Le secteur du bassin et la petite maison blanche}

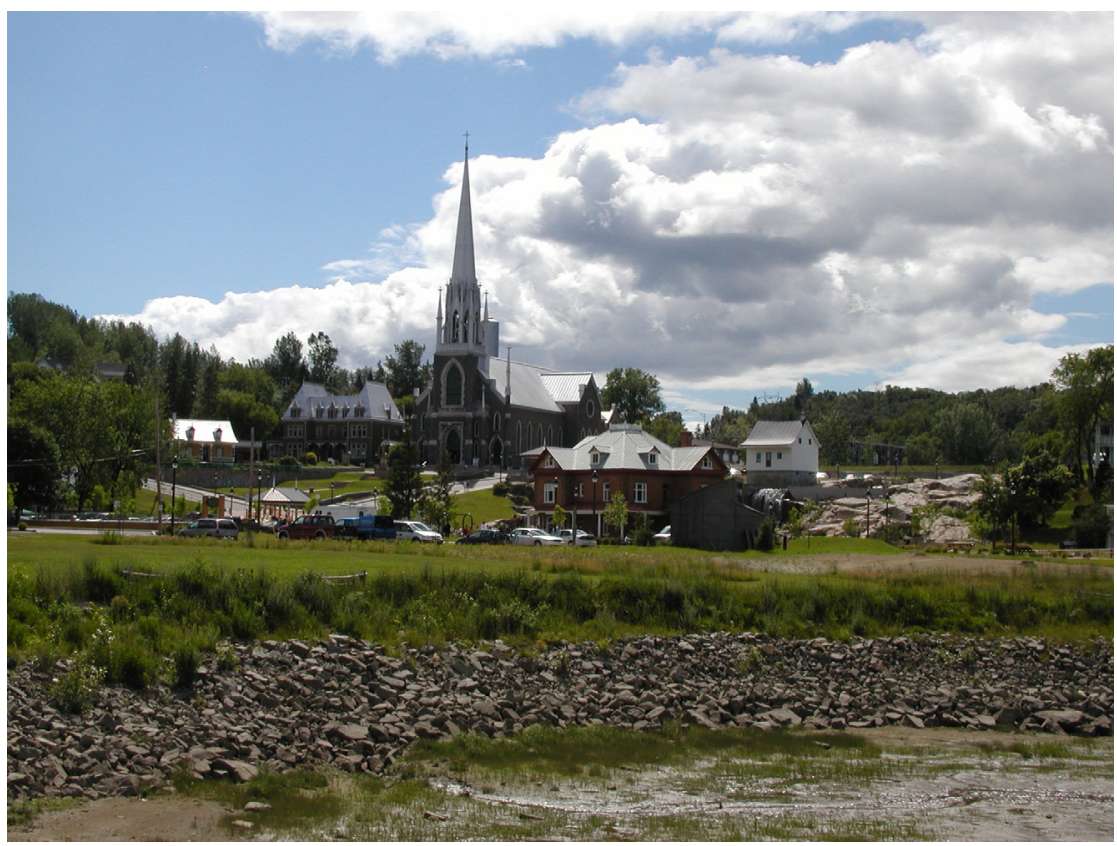

Source : Association des centres-villes de Chicoutimi

\section{Structure organisationnelle et cheminement du projet}

Afin de procéder à la formation du comité de travail $\mathrm{du}$ réseau du Croissant culturel et touristique, une assemblée regroupant la majorité des intervenants institutionnels et commerciaux de la zone a été convoquée en février 2003. La rencontre a permis de réaliser une consultation préliminaire auprès des participants, d'enrichir le concept de départ et de consacrer l'approche réseau adoptée par les instigateurs du projet. Un comité de travail a été formé afin d'accompagner l'Association des centres-villes de Chicoutimi dans sa démarche de développement du concept du Croissant culturel et touristique.

Le comité de travail devra conseiller l'Association des centres-villes dans l'élaboration d'initiatives découlant du développement du concept de base. À partir de buts et d'objectifs établis, d'inventaires et d'informations recueillies sur le milieu, le comité devra donner des avis techniques ou généraux sur l'orientation du projet. Il devra également s'assurer que les besoins du milieu soient pris en compte dans l'élaboration et la mise en œuvre du plan de développement, de même qu'en ce qui concerne la mise en valeur et la promotion du Croissant culturel et touristique de l'arrondissement de Chicoutimi.

La première réunion du réseau du Croissant culturel et touristique a eu lieu le 10 mars 2003. Ce fut une réunion extrêmement positive aux yeux de la plupart des participants. Le représentant de l'Association des centres-villes de Chicoutimi a expliqué les fondements du projet de Croissant culturel et touristique. Les intervenants présents ont également profité de cette première rencontre pour se donner une structure de fonctionnement et procéder à la nomination d'un secrétaire, d'un président et d'un vice-président du comité. Le principe de départ qui guide le comité est le suivant : que les arts et la culture soient de plus en 
plus visibles au centre-ville de l'arrondissement de Chicoutimi.

Depuis cette première rencontre, quatorze réunions du comité du Croissant culturel et touristique se sont tenues et six projets ont été réalisés :

1) colloque sur l'imaginaire urbain et la ville à l'UQAC (19 et 20 mars 2003);

2) rallye piétonnier patrimonial « $\mathrm{A}$ la découverte du Croissant culturel » (3 mai 2003);

3) lancement du dépliant promotionnel du Croissant culturel et touristique (12 juin 2003);

4) projets Arts-affaires (17 septembre au 9 octobre 2003) :

- vitrines artistiques au centre-ville (septembre et octobre 2003);

- visites commentées des vitrines lors des Journées de la culture (27 septembre 2003);

- prix du public en collaboration avec RadioCanada (8 octobre 2003 );

5) mise au point d'une signalétique et de repères visuels caractérisant le Croissant culturel et touristique (janvier 2004);

6) ciné-parc urbain sur la rue Racine dans le cadre du Festival «REGARD sur le court-métrage au Saguenay » (du 26 au 29 février 2004).

Tous ces projets ont servi à faire la promotion du Croissant culturel et touristique et ont donné des résultats à court terme sur le plan de la mobilisation des acteurs locaux, notamment des commerçants et du milieu artistique. La suite des choses prendra forme à l'intérieur du plan directeur à mesure que les besoins seront identifiés et que les objectifs seront définis et approuvés. La ville de Saguenay et le ministère de la Culture et des Communications constituent des partenaires de premier ordre dans le projet global de mise en valeur du Croissant. Ceux-ci appuient la revitalisation de cette zone en misant sur ses atouts culturels et patrimoniaux. De plus, la toute nouvelle politique culturelle de Saguenay tient compte du CCT en identifiant, à l'intérieur de son plan d'action, la valorisation du patrimoine et du cadre urbain dans cette zone. Dans cette optique, les arts et la culture pourraient devenir une source de développement tou- ristique pour l'ensemble du centre-ville et de l'arrondissement de Chicoutimi.

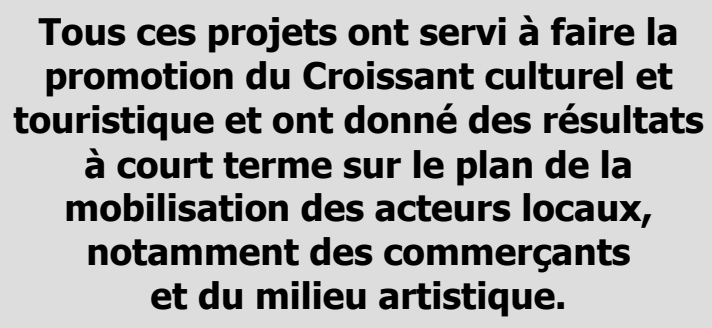

\section{Les défis associés au projet de Croissant culturel et touristique}

\section{Les particularités du territoire}

$\mathrm{Au}$ plan territorial, plusieurs défis confrontent les promoteurs du projet. Premièrement, le Croissant culturel et touristique couvre une très grande superficie, ce qui s'affiche comme un obstacle par rapport à l'unité qu'on veut donner à ce territoire en termes d'aménagement et de paysages. Deuxièmement, les trois pôles attractifs qui le composent et que nous avons décrits précédemment ne sont pas reliés entre eux par des réseaux de communication efficaces et balisés. À cet égard, le boulevard Saint-Paul se présente comme une ligne de discontinuité entre le quartier du Bassin et le reste du Croissant. Dans l'ensemble, il faudrait entrevoir le développement du Croissant culturel et touristique comme un quartier intégré, tout en misant sur l'amélioration du cadre de vie et la consolidation de la dynamique d'ensemble du milieu.

Pour ce faire, voici quelques objectifs qui pourraient servir de fondement au plan directeur du Croissant culturel et touristique et ainsi permettre de mieux insérer le concept dans le développement du centreville :

1) intégrer la dimension culturelle et touristique dans toute démarche d'intervention au centre-ville;

2) promouvoir le centre-ville en tant qu'espace artistique et culturel;

3) insérer le Croisant culturel et touristique dans les stratégies de promotion touristique de l'arrondissement de Chicoutimi et de la ville de Saguenay;

4) élaborer et mettre en œuvre un programme d'actions coordonnées par un plan d'aménagement; 
5) consolider la nouvelle vocation du secteur en favorisant la diversité des activités dans le domaine des arts, de la culture et du patrimoine.

\section{La participation des résidents}

Comme nous pouvons le constater, le projet de revitalisation et de dynamisation du centre-ville de l'arrondissement de Chicoutimi par le biais des arts et de la culture est bien lancé. La mobilisation des acteurs institutionnels, des gens d'affaires ainsi que des artistes et artisans nous permet d'être optimistes face à l'avenir. Cependant, l'implication des résidentes et résidents du secteur est un aspect important à favoriser au cours des prochains mois. Les citoyens demeurant à l'intérieur et à proximité du Croissant culturel et touristique doivent être associés à ce projet qui risque de transformer leur cadre de vie.

Une enquête réalisée par l'Association des centresvilles de Chicoutimi a permis de mieux saisir les attentes des populations directement concernées ${ }^{22}$. Cette enquête nous révèle que $63,3 \%$ des citoyens interrogés sont d'accord avec le projet de développement $\mathrm{du}$ visage culturel du centre-ville, mais affichent certaines craintes. Les craintes en question touchent la capacité réelle de réaliser un tel projet et, dans certains cas, quelques préjugés par rapport à la qualité ou au caractère élitiste des produits artistiques. Même s'ils connaissent peu le projet, la plupart des personnes interviewées se sont montrées sympathiques à la revitalisation du centre-ville, tout en constatant que celui-ci est dans une situation difficile sur un plan commercial, surtout lorsqu'on le compare au secteur du boulevard Talbot.

\section{Une enquête réalisée par l'Association des centres-villes de Chicoutimi nous révèle que $63,3 \%$ des citoyens interrogés sont d'accord avec le projet de développement du visage culturel du centre-ville, mais affichent certaines craintes.}

\section{La consolidation d'une culture urbaine}

Au-delà des résidents des quartiers anciens, il faudra faire en sorte que la population de l'ensemble de l'arrondissement s'approprie le Croissant en tant qu'espace collectif à fréquenter régulièrement. Dans le contexte actuel de développement commercial en milieu périurbain, les secteurs centraux trouvent une nouvelle vocation comme lieu ludique et festif ${ }^{23}$. Cette nouvelle fonction est reliée au caractère symbolique et historique du milieu. Les centres-villes sont généralement des lieux collectifs denses et animés qui se distinguent de l'aspect semi-privé des secteurs résidentiels pavillonnaires.

Même si les centres-villes n'ont pas la popularité d'antan, ils demeurent caractérisés par la présence d'espaces publics et de lieux de rassemblement. Dans l'arrondissement de Chicoutimi, le parc de la zone portuaire et la rue Racine constituent de vibrants exemples d'espaces de sociabilité que la population s'est appropriée. C'est dans ces endroits que se déroulent les activités festives comme que le Carnavalsouvenir ou le Festival de musique du monde. Il faut ajouter à cela les lieux de rencontres divers tels que la bibliothèque, les cafés, les bars et les restaurants. La fonction culturelle, artistique et patrimoniale pourrait devenir l'élément catalyseur d'une vie urbaine actuellement à l'état de latence.

Plusieurs pistes sont à exploiter pour établir une tradition de tourisme urbain à Chicoutimi. Par exemple, il serait possible d'implanter, à faibles coûts, des parcours patrimoniaux ou des visites d'ateliers d'artistes. Les établissements artistiques sont nombreux et répartis sur l'ensemble du territoire du Croissant. Dans le seul secteur du Bassin, on remarque les Ateliers TOUTTOUT, Le Lobe, La Chasse-Galerie, Caravane Films, Place du Presbytère, La Pulperie, Multi-art et les Ateliers Gauche-Droite.

\section{Conclusion}

À travers la mise en place du Croissant culturel et touristique, l'Association de centres-villes de Chicoutimi et les autorités municipales de Saguenay veulent signifier le rôle prépondérant des arts, de la culture et du patrimoine pour le développement économique et social du centre-ville de l'arrondissement de Chicoutimi. Ainsi, la mise en valeur des arts, de la culture et du patrimoine est maintenant considérée essentielle au maintien d'une dynamique urbaine vivante et attractive. Ce mouvement de réappropriation culturelle de l'urbain et de mise en exergue de la qualité de vie existe déjà dans les métropoles, et il semble en voie de s'implanter au sein des villes moyennes. 
S'inscrivant à l'intérieur de cette tendance, le projet $\mathrm{du}$ Croissant culturel et touristique de l'arrondissement de Chicoutimi est porteur d'avenir. Il s'agit d'un concept original qui se fonde sur une vision holistique du développement urbain et qui tente de tirer des leçons des faiblesses des programmes précédents. En effet, la revitalisation urbaine est une intervention publique dans le processus de développement urbain qui ne réussit pas toujours à canaliser les forces du marché dans la direction souhaitée. On doit fréquemment initier un nouveau plan d'intervention après quelques années. Dans un contexte où les ressources des administrations publiques sont relativement rares, il faut sortir de ce cercle vicieux et permettre aux espaces centraux de s'afficher dans ce qui les caractérise le mieux : leur atmosphère urbaine et la diversité des activités. De telles interventions axées sur les arts et la culture pourraient permettre d'améliorer la qualité de vie des résidents de l'arrondissement de Chicoutimi, et de Saguenay dans son ensemble. La croissance du tourisme urbain permettrait aussi une diversification des activités de loisirs au sein de cette ville.

\section{Ce mouvement de réappropriation culturelle de l'urbain et de mise en exergue de la qualité de vie existe déjà dans les métropoles, et il semble en voie de s'implanter au sein des villes moyennes.}

Selon une perspective éthique de l'urbanisme, la revitalisation du centre-ville de Chicoutimi se présente également comme un enjeu social, soit celui de fournir aux habitants des quartiers centraux un environnement et des équipements de même niveau que celui de l'ensemble des citoyens. D'autre part, la mise en œuvre d'une démarche de revitalisation par le biais des arts et de la culture constitue un défi à la capacité de gouvernance des administrateurs de la nouvelle ville et des intervenants locaux. Sommes-nous en mesure de réaliser des projets novateurs qui procurent une plus-value significative aux résidents et qui démontrent que Saguenay fait réellement partie des agglomérations québécoises au sein desquelles il fait bon vivre?

\section{Notes et références}

1 Martin Simard est professeur au département des sciences humaines et membre du Centre de recherche sur le développement territorial (CRDT) et du Centre interuni- versitaire d'études sur les lettres, les arts et les traditions (CÉLAT) de l'Université du Québec à Chicoutimi.

2 Nadine Maltais est agente de développement culturel à l'Association des centres-villes de Chicoutimi.

3 Les auteurs remercient Martin Dion de l'équipe de l'Atlas électronique du Saguenay-Lac-Saint-Jean pour l'aide apportée dans les traitements statistiques et cartographiques.

4 Filion, P., H. Hoernig, T. Bunting. et G. Sands (2003). «The Successful Few. Healthy Core Areas of Small Metropolitan Regions », in F. Charbonneau, P. Lewis et C. Manzagol (dir.), Villes moyennes et mondialisation. Pour un renouvellement des approches et des stratégies, Montréal, Trames, p. 101-117.

5 Porter, M. (1997). « New Strategies for Inner-City Economic Development», Economic Development Quaterly, vol. $11, \mathrm{n}^{\circ} 1$, p. 11-27.

6 Simard, M. et M.-J. Gauthier (2004). « Les enjeux territoriaux associés à la réforme municipale. L'exemple de Saguenay », Cahiers de géographie du Québec, vol. 48, $\mathrm{n}^{\circ} 134$ (à paraître).

7 Ce déménagement serait en grande partie lié aux politiques de localisation de la compagnie General Motors plutôt que d'être strictement tributaire de facteurs locaux.

8 Perron, M., L. Richard et S. Veillette (1997). « Structure sociorésidentielle et conditions de vie au Saguenay », Cahiers de géographie du Québec, vol. 41, n 112, p. 3148.

9 Speck, D. (2001). « Town or Sprawl?», dans J. Vaillancourt, A. Bourget et M. Ouellet (dir.), Vers des collectivités viables. Mieux bâtir nos milieux de vie pour le XXI siècle, Québec, Groupe Vivre en ville, Septentrion, p. 121-130.

10 Simard, M. (2000). «Développement local et identité communautaire : l'exemple du quartier Saint-Roch à Québec », Cahiers de Géographie du Québec, vol. 45, n 122, p. 167-188.

11 Bouchard, L.-M. (1973). Les villes du Saguenay : étude géographique, Chicoutimi, Leméac.

12 Wolfe, J.M. (1994). « Retour sur le passé : un survol historique de l'urbanisme canadien », Plan Canada, p. 1034.

13 Biddulph, M. (2000). «Re-Humanising Critiques of Suburbia », Urban Design Review, vol. 6, p. 159-172.

14 Binet, C. (2004). « Le contrôle de l'étalement urbain au Québec. 25 ans de valse-hésitation », Organisations et territoires, vol. 13, $\mathrm{n}^{\circ} 1, \mathrm{p} .83-91$.

15 Boudreault, P.W. (1986) Luttes régionalitaires et société post-industrielle. Dix ans d'actions socio-politiques au Saguenay, Jonquière, Sagamie/Québec. 
Séguin, A.-M. et G. Divay (2002). Pauvreté urbaine. La promotion de communautés viables, Ottawa, Réseaux canadiens de recherche en politiques publiques (RCRPP), document F27, 39 pages.

17 Bacqué, M.-H., G. Divay, D. Rose, A.-M. Séguin et G. Sénécal (2003). Survol de quelques politiques de revitalisation urbaine, Montréal, INRS-UCS, 102 p.

18 On parle des centres-villes de Chicoutimi au pluriel, car l'association inclut aussi les marchands de la rue Roussel sur la rive nord de la rivière Saguenay, dans l'ancienne municipalité de Chicoutimi-Nord. Nous nous concentrerons sur la portion sud du centre-ville à l'intérieur de ce texte.

19 Ce chiffre provient du recensement de 2001 de Statistique Canada. Il résulte d'une compilation spéciale de la popula- tion de 77 îlots urbains correspondant globalement aux frontières du Croissant. La population du secteur est en baisse. En effet, celle-ci était de 5105 habitants en 1976.

Bouchard, L.-M. (1973). Les villes du Saguenay : étude géographique, Chicoutimi, Leméac.

21 Les Verts Boisés du Fjord (1998). La trousse du marcheur Chicoutimi : un pas vers la santé!

22 Sasseville, M. (2003). Enquête sur les attentes de la population relativement au projet de Croissant culturel et touristique de l'arrondissement Chicoutimi, rapport de recherche, Saguenay, Association des centres-villes de Chicoutimi.

23 Fortin, A., C. Després et G. Vachon (dir.) (2002). La banlieue revisitée, Québec, Nota Bene. 


\section{Publicité}

\section{Revue Organisations et territoires}

« Site Web » 